\title{
Efeito de Terbufos em Soqueira sobre Fitonematóides Ectoparasitos de Cana-de-Açúcar
}

\author{
Andréa Chaves ${ }^{1}$, Elvira M. R. Pedrosa ${ }^{2} \&$ Romero M. Moura ${ }^{2}$ \\ ${ }^{1}$ Estação Experimental de cana-de-açúcar do Carpina, Rua Ângela Cristina C. P. de Luna. S/N, Bairro Novo-Carpina/PE, e- \\ mail: achavesfiuza@bol.com.br; ${ }^{2}$ Departamento de Agronomia, Universidade Federal Rural de Pernambuco, Dois Irmãos, \\ 52171-900, Recife, PE, e-mail: epedrosa_ufrpe@yahoo.com.br
}

(Aceito para publicação em 11/12/2002)

Autor para correspondência: Elvira Maria Régis Pedrosa

CHAVES, A., PEDROSA, E.M.R. \& MOURA, R.M. Efeito de terbufos em soqueira sobre fitonematóides ectoparasitos de cana-de-açúcar. Fitopatologia Brasileira 28:195-198. 2003.

\section{RESUMO}

Por ser comum a ocorrência de altos níveis populacionais de nematóides ectoparasitos em canaviais da região Nordeste, estudou-se o efeito do nematicida Terbufos (Counter 50G), em soqueira, em área infestada naturalmente por Helicotylenchus dihystera, Criconemella ornata e Paratrichodorus minor. O delineamento estatístico adotado foi o de blocos ao acaso em arranjo fatorial $2 \times 3 \times 5$ (duas épocas de corte $\mathrm{x}$ três tratamentos nematicida $x$ cinco variedades de cana-de-açúcar), com cinco repetições. Os tratamentos nematicidas consistiram de $3 \mathrm{~kg}$ i.a./ha aplicado por ocasião do plantio e após o primeiro corte, $4 \mathrm{~kg}$ i.a./ha aplicado no plantio, e testemunha, $0 \mathrm{~kg}$ i.a./ha, distribuídos em microparcelas de $2,0 \mathrm{~m} \times 2,0 \mathrm{~m}$. As avaliações, fundamentadas nas densidades populacionais dos nematóides, foram realizadas em cinco variedades de cana-de-açúcar (Saccharum spp.), CB45-3, RB813804, SP784764, SP79-1011 e SP 70-1143, na colheita da cana planta, 16 meses após o plantio, e primeira soca, 16 meses após o primeiro corte. Não houve interação entre nematicida, variedade e época de corte. As aplicações do nematicida e as variedades de cana não afetaram significativamente os níveis populacionais dos ectoparasitos. Entretanto, por ocasião do segundo corte, as densidades populacionais de $C$. ornata e $P$. minor foram significativamente menores do que no primeiro corte, ocorrendo o inverso com a população de $H$. dihystera.

Palavras-chave adicionais: controle químico, Paratrichodorus, Criconemella, Helicotylenchus, dinâmica populacional.

\begin{abstract}
Effect of Terbufos on plant ectoparasitic nematodes in sugarcane first ratoon

Due to the usually high population levels of ectoparasitic nematodes associated with sugarcane (Saccharum spp.) Northeastern Brazil, the effect of the systemic nematicide Terbufos (Counter 50G) was studied, on first ratoon, in soil naturally infested with Helicotylenchus dihystera, Criconemella ornata and Paratrichodorus minor. The experimental design consisted of completely randomized blocks with treatments factorially arranged in $2 \times 3 \times 5$ (two harvest periods $\mathrm{x}$ three nematicide doses $\mathrm{x}$ five sugarcane varieties), with five replicates. Nematicide doses

consisted of $3 \mathrm{~kg}$ a.i./ha, applied at planting and immediately after first cutting, $4 \mathrm{~kg}$ a.i./ha, applied at planting and control, $0 \mathrm{~kg}$ a.i./ ha, distributed into $2.0 \mathrm{~m}$ x $2.0 \mathrm{~m}$ microplots. Evaluations based on nematode population densities were carried out on five varieties, CB45-3, RB813804, SP78-4764, SP79-1011 e SP 70-1143, at plant and first ratoon harvest. There was no interaction among nematicide, variety and harvest time. Results showed nematicide doses and sugarcane varieties did not affect significantly nematode population levels. However, in contrast to $H$. dihystera, population densities of $C$. ornata and $P$. minor were significantly lower at ratoon than plant harvest.
\end{abstract}

ABSTRACT

Cultivada como monocultura contínua, a renovação dos campos de cana-de-açúcar (Saccharum spp.) quase sempre ocorre sem pousio entre remoção de soqueiras velhas e replantio. Essas condições favorecem o desenvolvimento de populações de fitonematóides (Spaull \& Cadet, 1990) e, como conseqüência, 275 espécies de 48 gêneros já foram registradas, associadas a essa gramínea, sendo os ectoparasitos os mais freqüentes (Novaretti et al., 1974; Moura \& Almeida, 1981; Maqbool \& Hashmin, 1987).

No Brasil, as primeiras referências de nematóides parasitando cana-de-açúcar, no Estado de São Paulo, datam de 1962 (Brieger, 1962), quando foram registrados os gêneros Helicotylenchus Steiner e Trichodorus Cobb, na variedade Co290. O primeiro levantamento de fitonematóides, feito através do convênio ESALQ-Copersucar, foi realizado no período de outubro de 1970 a abril de 1973, naquele Estado, verificando-se ocorrência do gênero Helicotylenchus em mais de $90 \%$ das 800 amostras coletadas (Novaretti et al., 1974).

Cruz et al. (1986), em levantamento populacional de nematóides em cana-de-açúcar em cinco unidades 


\section{A. Chaves et al.}

produtoras de Alagoas e Sergipe, observaram índices elevados dos gêneros Pratylenchus Graham, Helicotylenchus, e Criconemella De Grisse \& Loof, em contraste com baixos índices de Meloidogyne Goeldi e Trichodorus. Por outro lado, Moura et al. (2000), trabalhando com 1.097 amostras compostas de solo e raízes, coletadas em diversas áreas produtoras de cana-de-açúcar no Nordeste, relataram os gêneros Meloidogyne e Pratylenchus, como os mais freqüentes da região. Moura (2000) cita, afora os nematóides das galhas e das lesões, em seqüência de ocorrência, os gêneros Helicotylenchus, Paratrichodorus Siddiqi, Trichodorus, Tylenchorhynchus Cobb, Hemicycliophora De Man, Xiphinema Cobb, Radopholus Thorne, Longidorus (Micoletzky) Thorne \& Swanger e Rotylenchulus Linford \& Oliveira.

Embora amplamente distribuídos em regiões canavieiras, os nematóides do gênero Criconemella, são considerados de baixa severidade à cultura (Román, 1968). Pela severidade dos danos nas raízes, nematóides do gênero Trichodorus e Paratrichodorus são mais prejudiciais à cana-de-açúcar. Em experimentos na África do Sul, Trichodorus sp. e Paratrichodorus sp. restringiram absorção de água pelas raízes, limitando o crescimento dos colmos em cana planta; todavia, em Burkina Faso, oeste da África, menor efeito foi observado, porque a cana foi irrigada (Cadet, 1985). O mesmo autor observou que, em socaria, as perdas na África do Sul foram ocasionadas principalmente devido à presença de Xiphinema spp. e Paratrichodorus spp. Em Burkina Faso, os nematóides pouco interferiram na produtividade das socas, provavelmente, devido à predominância de Helicotylenchus dihystera (Cobb) Sher, considerado de baixa virulência à cultura da cana-de-açúcar. No Nordeste do Brasil, a influência de ectoparasitos na produtividade das socas e do controle químico na dinâmica populacional desses organismos têm sido pouco estudada, provavelmente, devido à ocorrência estar freqüentemente associada a endoparasitos de maior virulência.

O objetivo do presente trabalho foi avaliar, em soqueiras de cinco variedades de cana-de-açúcar, se o efeito de Terbufos aplicado no plantio se estenderia às socas e se haveria efeito cumulativo de uma aplicação no plantio e outra na soca sobre a dinâmica populacional de $H$. dihystera Criconemella ornata De Grisse \& Loof e Paratrichodorus minor (Colbran) Siddiqi, em área naturalmente infestada.

$\mathrm{O}$ experimento foi conduzido em microparcelas de 2,0 m x 2,0 m, constituídas por caixas de madeira de 30 $\mathrm{cm}$ de altura, ajustadas ao solo e afastadas entre si por uma distância de 1,5 m. A área escolhida foi de solo arenoso, altamente infestada por nematóides dos gêneros Meloidogyne, Pratylenchus, Helicotylenchus, Paratrichodorus e Criconemella, na Usina Santa Tereza PE. As variedades de cana-de-açúcar utilizadas foram CB45-3, RB813804, SP78-4764, SP79-1011 e SP70-1143. Um tratamento com Terbufos (Counter 50G) consistiu de
$60 \mathrm{~kg} / \mathrm{ha}$ (3 kg de i.a./ha), aplicado no plantio e após o primeiro corte; o segundo, $80 \mathrm{~kg} / \mathrm{ha}$ (4 kg de i.a./ha), aplicado no plantio; e testemunha ( $0 \mathrm{~kg}$ de i.a./ha). Nas socas, a aplicação do produto foi feita 30 dias após o corte da cana planta, cobrindo-se os tratamentos com solo do próprio leirão. As avaliações foram efetuadas em cana planta e soca. As densidades populacionais obtidas no corte da cana planta foram consideradas como as populações iniciais para efeito de comparação com as densidades populacionais nas socas. Com os três tratamentos nematicidas, as duas épocas de corte e as cinco variedades, formou-se um desenho experimental do tipo blocos ao acaso, em esquema fatorial, com cinco repetições. As populações dos ectoparasitos foram aferidas nas épocas de colheita da cana planta, 16 meses após o plantio, novembro de 1999, e 16 meses após, março de 2001, quando da colheita da primeira soca. Foram feitas médias de leituras dos índices populacionais dos nematóides em amostras de $300 \mathrm{~cm}^{3}$ de solo, processadas pelos métodos padrões (Jenkins, 1964; Barker, 1985). Os dados das estimativas populacionais dos fitonematóides para análise estatística foram transformados para $\log _{10}(\mathrm{x}+1)$.

Os resultados mostraram que não houve interação entre variedade, dosagem de Terbufos e época de corte em relação às densidades populacionais de nenhum dos gêneros estudados. Do mesmo modo, não foram significativas as interações de segundo nível nem os efeitos isolados de variedades e dosagens do nematicida (Tabela 1). Resultados semelhantes foram obtidos por Barros (2000), em cana planta, exceto para a combinação C. ornata x Terbufos (80kg/ha) x variedade SP70-1143, o que mostra ausência residual do nematicida no momento da colheita, favorecendo o restabelecimento das populações dos nematóides em todas as variedades estudadas. Roccia et al. (1975), trabalhando com Aldicarb e duas variedades de cana-de-açúcar, em área infestada com endo e ectoparasitos, observaram reduções das densidades populacionais dos fitonematóides, entretanto as avaliações foram realizadas seis meses após a aplicação do produto químico.

Segundo Novaretti \& Nelli (1980), a flutuação populacional de ectoparasitos como Helicotylenchus spp. e Trichodorus sp. depende diretamente das condições climáticas, principalmente precipitação e temperatura. Os dados do presente experimento mostraram que houve efeito significativo da época de corte sobre a densidade populacional das três espécies ectoparasitas estudadas (Tabela 1). As reduções nas densidades populacionais de $C$. ornata e $P$. minor podem ter favorecido a multiplicação de $H$. dihystera. Também, altas tempeOraturas e deficiências hídricas (Figura 1), nas condições em que se desenvolvem as socas no Nordeste, podem justificar menores populações dos ectoparasitos que têm o ciclo de vida inteiro na rizosfera. Mais pesquisas são necessárias para manejo mais eficiente de fitonematóides na região. 
Efeito de Terbufos em soqueira sobre fitonematóides ectoparasitos de cana-de-açúcar

TABELA 1 - Níveis populacionais de Criconemella ornata, Helicotylenchus dihystera e Paratrichodorus minor em cinco variedades de cana-de-açúcar (Saccharum spp.) tratadas com Terbufos, 16 meses após o plantio e primeiro corte. Médias de planta e soca por variedade e tratamento químico

\begin{tabular}{|c|c|c|c|c|c|c|c|c|c|c|}
\hline \multirow{3}{*}{ Variedade } & \multirow{3}{*}{$\begin{array}{c}\text { Dose de } \\
\text { Terbufos } \\
\text { (kg/ha) }\end{array}$} & \multicolumn{9}{|c|}{ Nematóide $/ 300 \mathrm{~cm}^{3}$ de solo* } \\
\hline & & \multicolumn{3}{|c|}{ Criconemella ornata } & \multicolumn{3}{|c|}{ Helicotylenchus dihystera } & \multicolumn{3}{|c|}{ Paratrichodorus minor } \\
\hline & & Planta & Soca & Média & Planta & Soca & Média & Planta & Soca & Média \\
\hline \multirow[t]{4}{*}{ SP70-1143 } & $0+0$ & 429 & 30 & 230 & 20 & 0 & 10 & 114 & 0 & 57 \\
\hline & $60+60$ & 136 & 0 & 68 & 17 & 0 & 8 & 95 & 0 & 47 \\
\hline & $80+0$ & 86 & 32 & 59 & 28 & 0 & 14 & 108 & 0 & 54 \\
\hline & & 217 & 21 & & 22 & 0 & & 106 & 0 & \\
\hline \multirow[t]{4}{*}{ RB813804 } & $0+0$ & 276 & 0 & 138 & 9 & 64 & 36 & 114 & 0 & 57 \\
\hline & $60+60$ & 314 & 32 & 173 & 20 & 64 & 42 & 133 & 32 & 82 \\
\hline & $80+0$ & 259 & 127 & 193 & 7 & 32 & 20 & 67 & 0 & 34 \\
\hline & Média & 283 & 53 & & 12 & 53 & & 105 & 11 & \\
\hline \multirow[t]{4}{*}{ SP78-4764 } & $0+0$ & 228 & 0 & 114 & 34 & 64 & 40 & 134 & 0 & 67 \\
\hline & $60+60$ & 129 & 127 & 128 & 48 & 32 & 69 & 65 & 0 & 33 \\
\hline & $80+0$ & 75 & 0 & 37 & 11 & 127 & 49 & 95 & 0 & 47 \\
\hline & Média & 144 & 42 & & 31 & 74 & & 98 & 0 & \\
\hline \multirow[t]{4}{*}{ CB45-3 } & $0+0$ & 168 & 32 & 100 & 30 & 32 & 31 & 125 & 32 & 78 \\
\hline & $60+60$ & 358 & 64 & 211 & 6 & 0 & 3 & 119 & 31 & 75 \\
\hline & $80+0$ & 306 & 32 & 169 & 77 & 0 & 38 & 218 & 0 & 109 \\
\hline & Média & 277 & 42 & & 37 & 11 & & 154 & 21 & \\
\hline \multirow[t]{4}{*}{ SP79-1011 } & $0+0$ & 97 & 95 & 96 & 13 & 95 & 54 & 103 & 0 & 51 \\
\hline & $60+60$ & 207 & 95 & 151 & 11 & 159 & 85 & 151 & 0 & 76 \\
\hline & $80+0$ & 106 & 95 & 101 & 32 & 127 & 79 & 108 & 0 & 54 \\
\hline & Média & 137 & 95 & & 19 & 127 & & 121 & 0 & \\
\hline Média Geral & & $212 \mathrm{~A}$ & $51 \mathrm{~B}$ & & $24 \mathrm{~B}$ & $53 \mathrm{~A}$ & & 117A & $6 \mathrm{~B}$ & \\
\hline
\end{tabular}

* Dados transformados em $\log _{10}(\mathrm{x}+1)$ para análise estatística e apresentados sem transformação. Interação entre variedade, dose de Terbufos e época de corte não significativa. Para o mesmo nematóide, na mesma linha, médias de planta e soca seguidas por diferentes letras maiúsculas diferem estatísticamente ao nível de $5 \%$ de probabilidade pelo teste de Tukey.

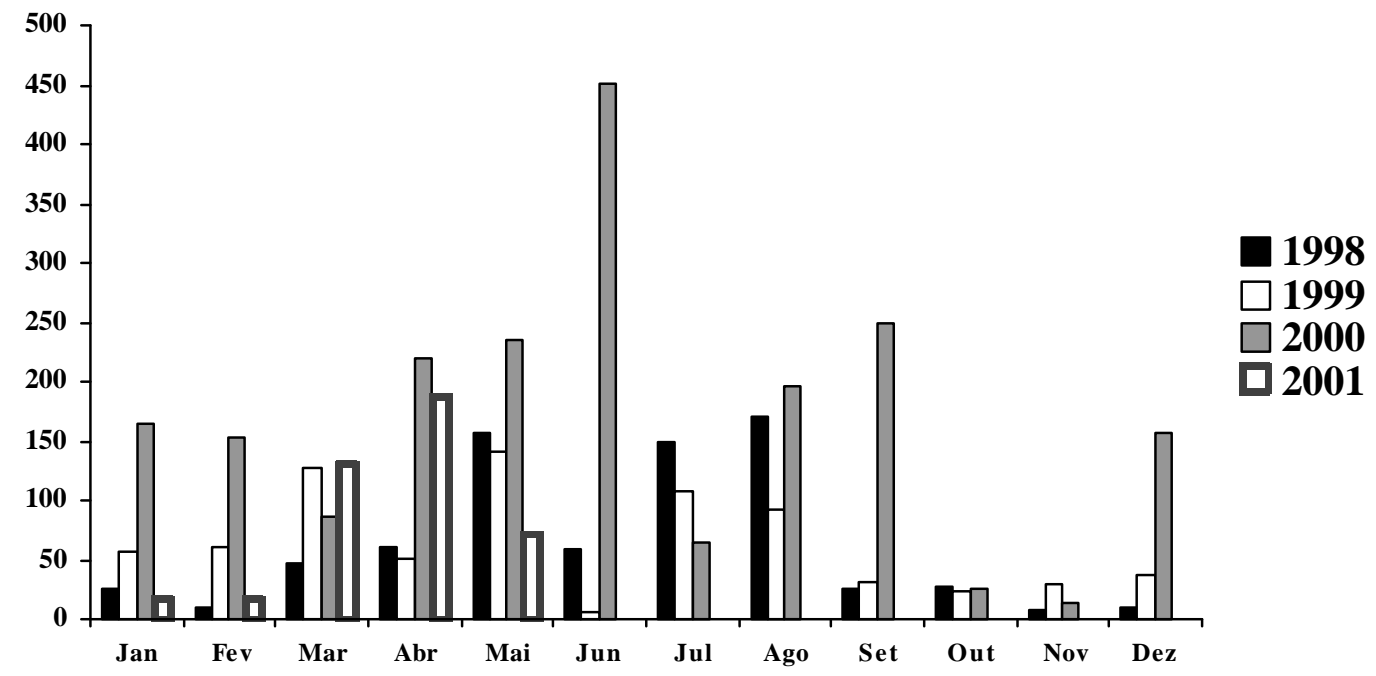

FIG. 1 - Índices de precipitação pluviométrica anual aferida na Usina Santa Tereza (1998-2001).

\section{REFERÊNCIAS BIBLIOGRÁFICAS}

BARKER, K.R. Sampling nematode communities. In: Barker, K.R., Carter, C.C. \& Sasser, J.N. (Eds.) An advanced treatise on Meloidogyne, v.2. Methodology. Raleigh. North Carolina State University Graphics. 1985. pp.3-17.

BARROS, A.C.B. Estudos sobre o uso do nematicida sistêmico Terbufos em cana-de-açúcar: avaliações na cana planta. (Dissertação de Mestrado). Recife. Universidade Federal Rural de Pernambuco. 2000.
BRIEGER, F.A. Recomendações para o plantio da cana-de-açúcar. São Paulo. Cooperativa Oeste do Estado de São Paulo, Boletim n.10. 1962.

CRUZ, M.M., SILVA, S.M.S. \& RIBEIRO, C.A.G. Levantamento populacional de nematóides em cana-de-açúcar em áreas de baixa produtividade nos Estados de Alagoas e Sergipe. Nematologia Brasileira 10:27-28. 1986.

CADET, P. Incidence des nèmatodes sur les reponsses de canne à sucre au Burkina Faso et en Côte d'Vore. Revue de Nèmatologie 8:277-284. 1985 


\section{A. Chaves et al.}

JENKINS, W.R. A rapid centrifugal-flotation technique for separating nematodes from soil. Plant Disease Reporter 48:692. 1964.

MAQBOOL, M.A. \& HASHMIN, S. Effect of granular nematicides on nematode populations and sugarcane yield. Revue de Nématologie 10:111-113. 1987.

MOURA, R.M. Controle integrado dos nematóides da cana-deaçúcar no nordeste do Brasil. Anais, $22^{\circ}$ Congresso Brasileiro de Nematologia, Uberlândia, MG. 2000. pp.88-94.

MOURA, R.M. \& ALMEIDA, A.V. Estudos preliminares sobre a ocorrência de fitonematóides associados à cana-de-açúcar em áreas de baixa produtividade agrícola no Estado de Pernambuco. Anais, $5^{a}$ Reunião Brasileira de Nematologia, Piracicaba, SP. 1981. pp.213220.

MOURA, R.M., PEDROSA, E.M.R., MARANHÃO, S.R.V.L., MACEDO, M.E.A., MOURA, A.M., SILVA, E.G. \& LIMA, R.F. Ocorrência dos nematóides Pratylenchus zeae e Meloidogyne spp. em cana-de-açúcar no Nordeste do Brasil. Fitopatologia Brasileira 25:101-103. 2000.
NOVARETTI, W.R.T. \& NELLI, E.J. Flutuação populacional de nematóides na cultura da cana-de-açúcar - cana de ano e meio. Brasil Açucareiro 96:30-36. 1980.

NOVARETTI, W.R.T., ROCCIA, A.O., LORDELLO, L.G.E. \& MONTEIRO, A.R. Contribuição ao estudo dos nematóides que parasitam a cana-de-açúcar em São Paulo. Anais, $1^{a}$ Reunião Brasileira de Nematologia, Piracicaba, SP. 1974. pp.27-32.

ROCCIA, A.O., LORDELLO, L.G.E. \& LORDELLO, R.R.A Ensaios de controle de nematóides em cana-de-açúcar com Aldicarb. Revista de Agricultura 50:175-181. 1975.

ROMÁN, J. Nematode problems of sugarcane in Puerto Rico. In: Smart Jr., G.C. \& Perry, V.G. (Eds.) Tropical Nematology. Gainesville. Publish Center for Tropical Agriculture, University of Florida Press. 1968. pp.61-67.

SPAULL, V.W. \& CADETT, P. Nematode parasites of sugarcane. In: Luc, M., Sikora R.A. \& Bridge, J. (Eds.) Plant parasitic nematodes in subtropical and tropical agriculture. Wallingford. C.A.B. International Institute of Parasitology, C.A.B. International. 1990. pp.461-491. 